\title{
Real-Time Optimization of an Active Filter's Performance
}

\author{
Simon D. Round, Student Member, IEEE, and Richard M. Duke, Member, IEEE
}

\begin{abstract}
Recent advances in power electronics have meant that many loads now draw a distorted current from the power supply. For the same real power consumed, the apparent power for the distorted load is greater than the equivalent sinusoidal load. A real-time active filter optimization algorithm has been implemented in a TMS320C30 DSP, with the aim of maximizing the monetary savings from active filtering by reducing the apparent power consumed at the point of supply. As the basis for this optimization a savings function which takes into account active filter efficiency, the cost of energy, and the supply and load current distortion before and after filtering, has been derived. $A$ simplex optimization technique, which is able to find the optimum operating point even under varying load conditions, is used to maximize these energy savings.
\end{abstract}

\section{INTRODUCTION}

$\mathbf{O}$ VER recent years there has been a proliferation of products containing some form of power electronic control [1]. These power electronic devices tend to make more efficient use of the available energy by converting it into a directly usable form. However, to minimize the capital cost of the conversion equipment, the designers often allow the power drawn from the mains supply to contain harmonic currents. These harmonic currents cause an increase in the level of rms supply current, which results in an increase in apparent power consumed at the point of supply. Nonsinusoidal supply currents can also cause overheating in supply transformers, communication interference, and excessive neutral currents [2]. It is now possible to efficiently use active filters to remove these harmonic components and overcome many of the disadvantages of passive filters [3], [4].

Extensive research has been carried out by many researchers on different converter configurations, switching control (modulation) techniques, and signal processing for active filter systems [5]-[8]. This paper however is concerned with maximizing the monetary savings gained from active filtering. This is achieved by reducing the apparent power consumed at the point of supply and considering the cost of the additional real power required to overcome the active filter losses.

The single phase active filter chosen for this purpose has been described elsewhere [9], [10]. A block representation of the active filter and signal processing unit (SPU) is detailed in Fig. 1. A synthetic fundamental sinusoid with adjustable phase delay is derived in the SPU from the nonlinear load current $I_{L}$. The synthetic sinusoid is subtracted from the distorted load current signal to produce a compensating current

Manuscript received July 23, 1993.

The authors are with the Department of Electrical and Electronic Engineering, University of Canterbury, Christchurch, New Zealand.

IEEE Log Number 9400212 .

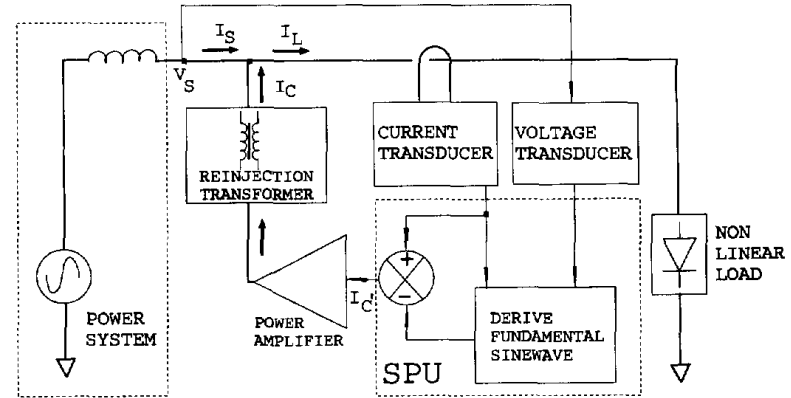

Fig. 1. Active filtering system.

signal. This compensating current signal contains the distortion components required to compensate for the supply current distortion caused by the load. The compensating current signal is then amplified by a controlled current, voltage source power amplifier, using a simple time delay switching strategy, to produce the required compensating current $I_{C}$. With the compensating current being reinjected into the power system the supply current $I_{S}$ becomes nearly sinusoidal. The main feature of this active filtering system is that the signal processing operates in the time domain and is not slowed by the need for complex signal processing to extract the harmonic frequency components.

This paper briefly discusses a digital implementation of the active filter control system, which is then used to implement a real-time optimization algorithm. The basis for this optimization is the development of a savings function which takes into account the efficiency of the active filter, the cost of energy, and the supply current distortion before and after filtering. The ultimate goal is to maximize the monetary savings and operating efficiency while minimizing supply current distortion.

\section{COST OF ENERGY}

Ideally, equipment connected to the power system should only consume real power. This however is not the case, some items of equipment draw a current component which is not converted into real power. These currents are defined in this paper as distortion currents and they can contain both harmonic and phase displaced components. The harmonic and displacement components can be defined by distortion $(\mu)$ and displacement $(\cos \theta)$ factors, respectively [2]. In the special case where the supply voltage is sinusoidal, the total power factor $\left(p_{f}\right)$ at the point of supply is the combination of 


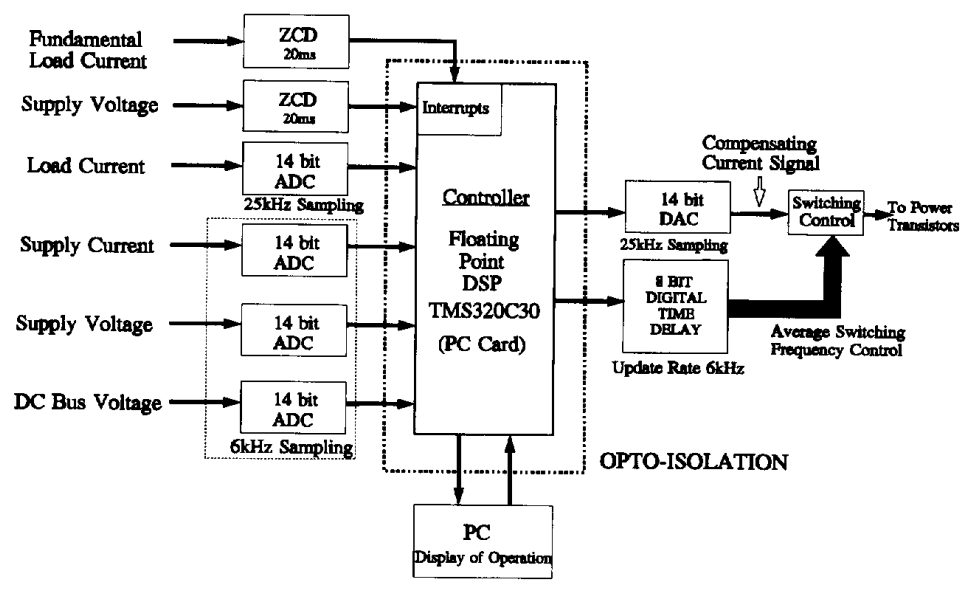

Fig. 2. Interfacing the active filter to the TMS320C30 DSP.

harmonic and displacement distortion as shown by (1).

$$
p_{f}=\mu \cos \theta \text {. }
$$

It can also be shown [11] that if $D_{\mathrm{TH}}$ is the Total Harmonic Distortion (THD) of the supply current, then

$$
\mu=\frac{1}{\sqrt{1+D_{\mathrm{TH}}^{2}}} \text {. }
$$

The charge for electrical energy consumed generally depends on the type of consumer. For domestic and small businesses, the charge is usually based only on the real power $(P)$ consumed per hour $(\mathrm{kWh})$. Industrial/commercial consumers' charges are however based on both the real power and the peak demand apparent power consumed. For a constant load this peak demand apparent power $(S)$ can be converted into an hourly rate (kVAh). An energy cost ratio, $R_{c}$, can be defined as the ratio of charge for real power $\left(c_{P}\right)$ to apparent power $\left(c_{S}\right)$ at some common time rate [(3)].

$$
R_{c}=\frac{c_{P}}{c_{S}}
$$

This energy cost ratio effects the total cost of energy to the consumer and can have a profound influence over the level of savings achievable by active filtering. Typically in New Zealand, real power is worth approximately five times that of apparent power, an $R_{c}$ of five. By decreasing the energy cost ratio, an additional cost can be added to penalize the consumer for distortion power consumed. Since the distortion components of a particular consumers' load will usually vary with time, an active filter therefore requires some form of intelligent controller to optimize the possible savings from active filtering, while taking into account the supply current distortion and the energy cost ratio.

\section{IMPLEMENTATION OF A DigITAL CONTROLLER}

The intelligent digital controller for the active filter is based on the TMS320C30 Digital Signal Processor (DSP) [12]. The controller's primary task is to measure the distorted load current, subtract a fundamental sinusoid, and output the resulting compensating current signal to the switching control circuit in real time. Interconnection of the TMS320C30 to the external interfaces of the active filter is shown in Fig. 2. 14 bit ADC's and DAC's give the system a large dynamic range. The sample rate for the load current ADC system was determined so that the delay between the input sample and the output of the computed result is less than one degree of phase delay at the fundamental frequency. For a supply frequency of $50 \mathrm{~Hz}$ this results in a sample rate greater than $18 \mathrm{kHz}$. A variable sample rate of about $25.6 \mathrm{kHz}$ is used so that exactly 512 samples are recorded during each fundamental period.

Supply current, supply voltage and power amplifier dc bus voltage ADC's (Fig. 2) and the time delay switching frequency control output are all sampled at the lower rate of $6.4 \mathrm{kHz}$, which corresponds to 128 samples per fundamental period. The average switching frequency of the active filter can be adjusted in the range from $2 \mathrm{kHz}$ to $80 \mathrm{kHz}$ and the dc bus voltage proportional-integral controller adjusts the magnitude of the synthetic sinusoid to control the real power flow into the active filter to overcome losses. A sample rate of $6.4 \mathrm{kHz}$ is sufficient for the 128-point FFT to calculate up to the 50th harmonic component, while allowing the TMS320C30 more time for the calculation of other control functions. Three 128point FFTs provide information so the operating efficiency of the active filter, power factor, and the THD level of the supply and load currents can be calculated.

\section{FILTER PERFORMANCE}

When the load current contains both harmonic and displacement distortion components the active filter can provide total power factor correction. This is demonstrated in Fig. 3 for a resistor and inductor combination in parallel with a single phase bridge rectifier with capacitive and resistive load. The phase displacement of the resistor and inductor combination is $80^{\circ}$ and the total load has a displacement of $56^{\circ}$ with a load current THD of $40.3 \%$, which results in a total power factor of 0.630 [using (1)]. When the active filter is operated the supply current THD is reduced to $9.1 \%$, with a displacement of $0^{\circ}$, and a resultant power factor of 0.991 . The supply current now 


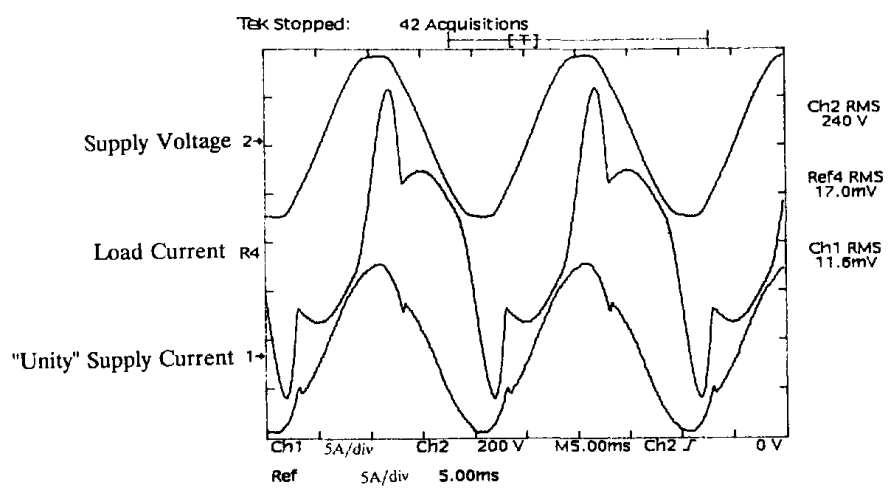

Fig. 3. Distortion and displacement compensation.

has a value of $5.8 A_{\mathrm{RMS}}$ compared to the load current value of $8.5 A_{\text {RMS }}$.

The operational performance of the active filter is determined by the efficiency with which it is able to reduce supply current distortion. Fig. 4 shows the efficiency of the active filter and the supply current THD for various values of dc bus voltage and average switching frequency. These results were obtained while the active filter was compensating for only the harmonic distortion component of load current (THD $=78 \%$ ) drawn by a single phase bridge rectifier with capacitive and resistive load. At high levels of dc bus voltage and average switching frequency the supply current THD is reduced the most, however at this point efficiency is at its lowest (87.9\%). It should be possible to optimize the operating conditions of dc bus voltage and average switching frequency with the aim of minimizing the supply current THD while maximizing efficiency. One approach is to derive a savings function which describes the operation of the active filter in terms of the actual financial cost of the total electricity consumption of the load plus active filter.

\section{SAVINGS FUNCTION}

A savings function can be defined as the difference between the cost for the energy consumed before and after the process of active filtering. An optimization system could then use this savings function to determine the most economic operating point. To determine the cost before and after active filtering, real and apparent power flow definitions (Fig. 5) must be considered. For the case where no active filtering is taking place the cost to the consumer for a load $\left(P_{L}, S_{L}\right)$ is defined by the cost function, $C_{\mathrm{NAF}}[(4)]$ where $p_{f \mathrm{NAF}}=P_{L} / S_{L}$.

$$
\begin{aligned}
C_{\mathrm{NAF}} & =P_{L} c_{P}+S_{L} c_{S} \\
& =P_{L} c_{P}\left(1+\frac{1}{R_{c} p_{f \mathrm{NAF}}}\right) .
\end{aligned}
$$

When active filtering is included, the extra power $(\Delta P)$ the active filter consumes, depends on the amount of compensation it is providing and the operational efficiency $(\zeta)$ of the filter. From the usual definition of efficiency, this extra power is

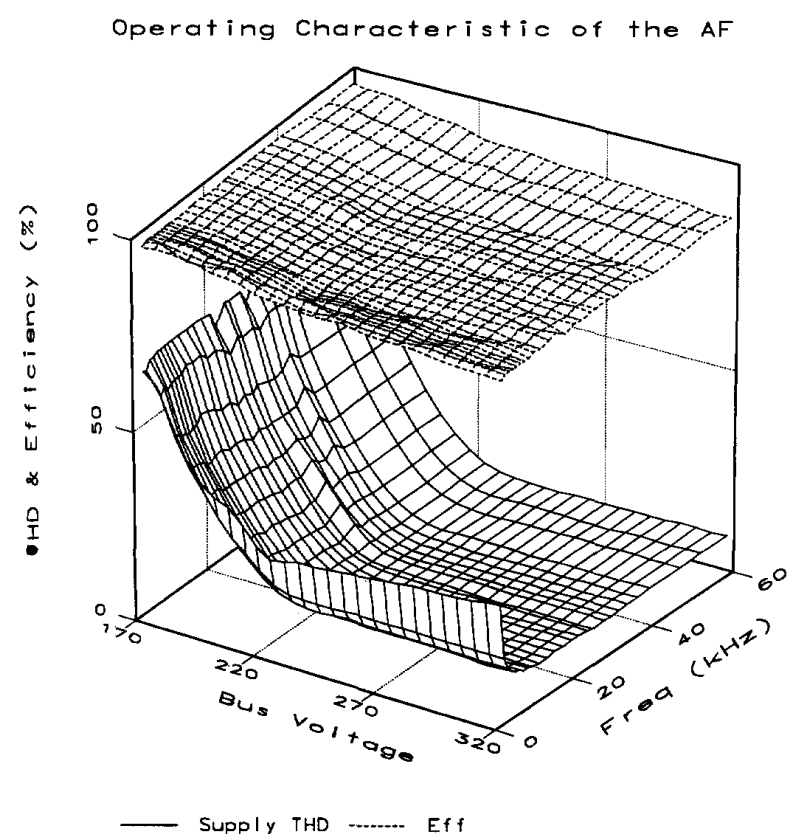

Fig. 4. Operating characteristic of the active filter

given by

$$
\Delta P=P_{L}\left(\frac{1-\zeta}{\zeta}\right)
$$

With the same nonlinear load and when active filtering is included, the kVA level at the point of supply has now changed $\left(S^{\prime}\right)$ and the real power consumption from the system has increased $\left(P^{\prime}\right)$, resulting in a new cost function, $C_{\mathrm{AF}}[(6)]$ where $p_{f \mathrm{AF}}=P^{\prime} / S^{\prime}$.

$$
\begin{aligned}
C_{\mathrm{AF}} & =P^{\prime} c_{P}+S^{\prime} c_{S} \\
& =P_{L} c_{P}\left(1+\left(\frac{1-\zeta}{\zeta}\right)+\frac{1}{R_{c} p_{f \mathrm{AF}}}+\frac{\left(\frac{1-\zeta}{\zeta}\right)}{R_{c} p_{f} \mathrm{AF}}\right)
\end{aligned}
$$




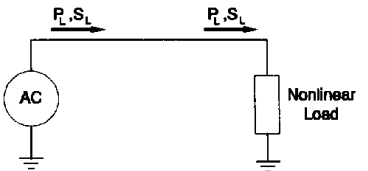

(a)

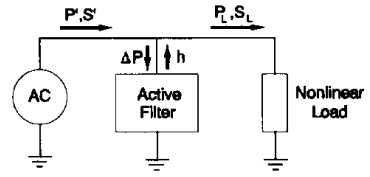

(b)
Fig. 5. Definition of power flow. (a) No active filtering. (b) Active filtering.

The savings $\left(s_{\mathrm{AV}}\right)$ made from active filtering can now be described by $(7)$.

$$
\begin{aligned}
s_{\mathrm{AV}}= & C_{\mathrm{NAF}}-C_{\mathrm{AF}} \\
= & P_{L} c_{P}\left(\frac{1}{R_{c} p_{f \mathrm{NAF}}}-\frac{1}{R_{c} p_{f \mathrm{AF}}}\right. \\
& \left.-\left(\frac{1-\zeta}{\zeta}\right)\left(1+\frac{1}{R_{c} p_{f \mathrm{AF}}}\right)\right) .
\end{aligned}
$$

By expressing these savings as a percentage of the cost of operating the load [(8)], it is possible to remove the dependency on the real power consumed by the load $\left(P_{L}\right)$ and the charge for that power $\left(c_{P}\right)$.

$$
\begin{aligned}
s_{\mathrm{AV}}(\%) & \frac{\frac{1}{R_{c} p_{f \mathrm{NAF}}}-\frac{1}{R_{c} p_{f \mathrm{AF}}}-\left(\frac{1-\zeta}{\zeta}\right)\left(1+\frac{1}{R_{c} p_{f \mathrm{AF}}}\right)}{1+\frac{1}{R_{c} p_{f \mathrm{NAF}}}} \\
& \times 100
\end{aligned}
$$

Utilizing (1) and (2), the percentage savings can now be expressed in terms of supply current THD and fundamental phase displacement as given by (9).

$$
\begin{aligned}
& s_{\mathrm{AV}}(\%) \\
& \quad \frac{\frac{\sqrt{1+D_{\mathrm{THNAF}}^{2}}}{R_{c} \cos \theta_{\mathrm{NAF}}}-\frac{\sqrt{1+D_{\mathrm{THAF}}^{2}}}{R_{c} \cos \theta_{\mathrm{AF}}}-\left(\frac{1-\zeta}{\zeta}\right)\left(1+\frac{\sqrt{1+D_{\mathrm{THAF}}^{2}}}{R_{c} \cos \theta_{\mathrm{AF}}}\right)}{1+\frac{\sqrt{1+D_{\mathrm{THNAF}}^{2}}}{R_{c} \cos \theta_{\mathrm{NAF}}}} \\
& \times 100(\%)
\end{aligned}
$$

Using the efficiency and THD information from Fig. 4 and a cost ratio of five, the savings gained from active filtering for the single phase bridge rectifier load are given in Fig. 6 . The maximum savings point in Fig. 6 has a value of $0.2 \%$ at a dc bus voltage of $189 \mathrm{~V}$ and an average switching frequency of 5 $\mathrm{kHz}$. At this maximum point the supply current THD is $41.2 \%$, compared to the load current THD of $78.0 \%$, and the active filtering system has an operating efficiency of $97.3 \%$. Thus, by simply maximizing savings a high operating efficiency has been achieved but the reduction in supply current THD has not been very substantial. It may be desirable to operate the active filtering system so as to achieve some minimum required level of supply current THD. The IEEE-519 harmonic standard for example specifies the required current THD at the point of common coupling [13]. Therefore a required level of supply current THD should be incorporated into the calculation of the savings surface. This can be achieved by adding a penalty to the savings function $\left(s_{\mathrm{AV}}(\%)\right)$ to ensure that the supply

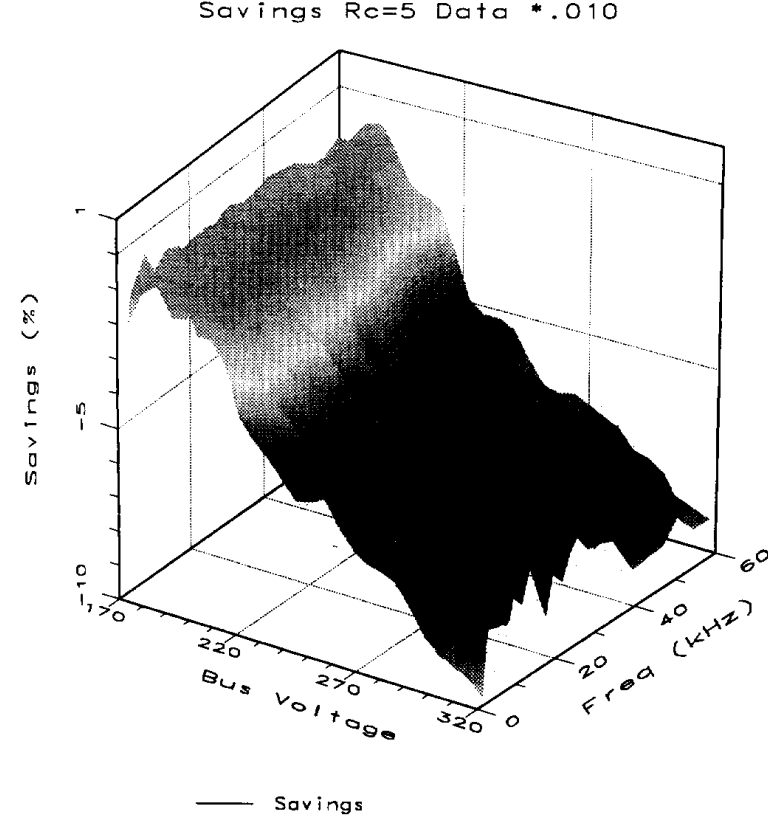

Fig. 6. Energy savings gained by active filtering.

current THD is lower than some set maximum ( $\left.D_{\mathrm{MAX}}\right)$ and can be described by the following expression:

$$
\begin{gathered}
s_{\mathrm{AV}}(\%)+\sigma D_{\mathrm{TH}} \mathcal{F}\left(D_{\mathrm{TH}}\right) \\
\text { where } \sigma<0 \\
\text { and } \mathcal{F}\left(D_{\mathrm{TH}}\right)=\left\{\begin{array}{ll}
1: & D_{\mathrm{TH}}-D_{\mathrm{MAX}}>0 \\
0: & D_{\mathrm{TH}}-D_{\mathrm{MAX}} \leq 0
\end{array} .\right.
\end{gathered}
$$

The penalty term, which only operates when the supply current THD ( $D_{\mathrm{TH}}$ ) is greater than $D_{\mathrm{MAX}}$, is proportional to the amount of supply current THD present. The scaling term $(\sigma)$ is negative so that the penalty function reduces the savings and forces the maximum savings point to a region where the supply current THD is less than that specified by $D_{\text {MAX }}$.

The effect of such a penalty function is shown in Fig. 7 . The savings curve (dotted lines) is for the same single phase bridge rectifier load described previously and an energy cost ratio of one. The maximum savings operating point without any penalty imposed is at $207 \mathrm{~V}$ and $5 \mathrm{kHz}$. At this point the efficiency of the active filtering system is $96 \%$ and the supply current THD is reduced from $78.0 \%$ to $23.4 \%$ (Fig. 4). However if $D_{\text {MAX }}$ is now set to $10 \%$ and a penalty is added using a scaling term $\sigma$ of -0.5 , a penalty surface (solid lines) is produced, also shown in Fig. 7. The shaded region in Fig. 7 is where no penalty is added to the savings surface because the supply current THD is less than $D_{\text {MAX }}$ and therefore the savings and penalty surfaces are identical. The maximum savings point for the supply current THD to be less than $10 \%$ occurs at a bus voltage of $231 \mathrm{~V}$ and switching frequency of $18 \mathrm{kHz}$. At this point the active filtering system operates with an efficiency of $92.8 \%$ and the supply current THD is reduced from $78.0 \%$ to $9.8 \%$. 


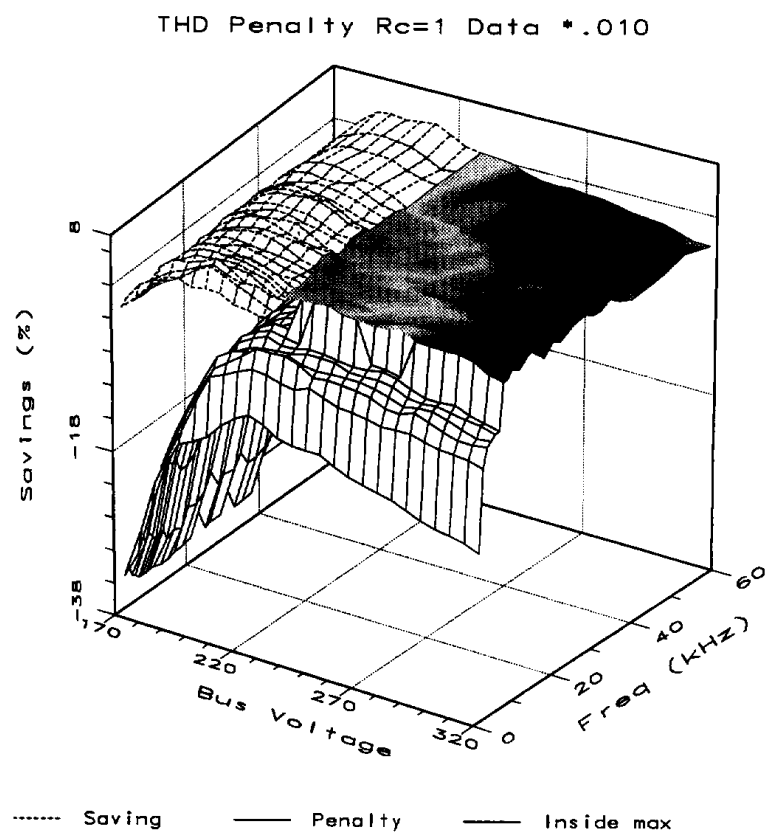

Fig. 7. Effect of setting maximum allowed THD.

\section{MAXIMIZATION OF SAVINGS}

A savings function, which calculates the possible savings available to the consumer by active filtering, has been derived. The active filter's operating conditions can be altered to achieve a maximum savings point. An automatic search procedure, which is independent of the type of load and $D_{\mathrm{MAX}}$ level, is necessary to find this maximum savings point. Since more than one operating condition can be altered to move about on the savings surface, a multidimensional optimization algorithm is required. Unlike the more traditional optimization problems in which the shape of the surface is known, the savings surface is unknown prior to active filtering. The surface is only measured when the optimization algorithm steps towards the maximum savings point and due to time varying loads this measured data is continually changing. Any optimization algorithm must therefore continue to explore the area surrounding the maximum savings point in order to find new maxima over time.

A direct method, which starts at an arbitrary point and proceeds in a stepwise fashion towards the maxima or minima by successive improvements, is an appropriate optimization procedure. Many different types of direct methods are available, however these all fall into two categories. Firstly, methods using the first derivative information such as the conjugate gradient and variable metric methods [14]. Secondly, methods that do not need to compute the first derivative, such as the direction-set and the simplex method [14]. Since the savings surface is unknown the use of first derivative methods require the operating point of the active filter to be altered for each measurement. The simplex method on the other hand moves towards the maxima in a more straightforward fashion that

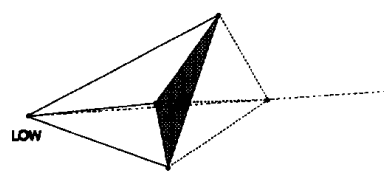

(a) $\mathrm{HIOH}$

(c)

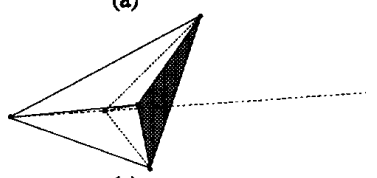

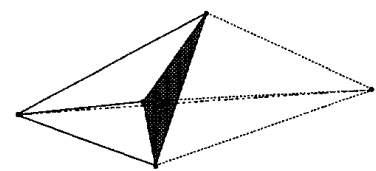

(b)

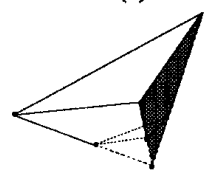

(d)
Fig. 8. Modes of the Simplex Algorithm.

makes no assumptions about the surface [14]. Although the simplex method tends to be slow it was used to find the optimum operating point because it can be very robust. The simplex method is based on using the geometric figure called a simplex, consisting of $N+1$ vertices (for $N$ dimensions). In two dimensions the simplex is a triangle and in three dimensions it is a tetrahedron.

The simplex method is started with a single starting point and the vertices of the initial simplex are calculated using this point and orthogonal unit vectors. The simplex method can take a series of four different steps towards the optima. These steps are graphically depicted in Fig. 8 for a threedimensional $(N=3)$ case [14]. Initially the measured data from the four starting points is sorted and the highest and lowest points are determined. The first step is taken by moving the lowest point through the opposite face of the simplex [see Fig. 8(a)]. This step is called a reflection. If this new point has a higher value then the reflection length is expanded [Fig. 8(b)]. If the new point is higher again then this point is accepted otherwise the previous point is used. The old low point is now disregarded. However if the first reflected point is worse than the second lowest point then the simplex will contract along one dimension [Fig. 8(c)]. If this contraction does not yield an improvement the simplex contracts itself in all directions, pulling itself in around its highest point [Fig. 8(d)]. The simplex algorithm terminates when the step towards the maxima is smaller than a preset tolerance value.

The simplex algorithm is however dependent on history terms (measured vertices) to calculate the next step towards the point of maximum savings. These history terms can cause a problem when the savings surface changes due to a new load condition, cost ratio or maximum supply current THD. If the savings surface rises due to such a change, the simplex algorithm will continue to try and find a new maximum and thus track the new surface. However in the case where the new savings surface is lower, the simplex algorithm will not find the new maximum because the history terms have not changed and they contain the old high values. To enable the simplex algorithm to follow these changes in the savings surface, the load current rms and THD values are measured. When a variation (presently 10\%) is detected in either term the simplex algorithm remeasures the history terms and starts the optimization search again. 


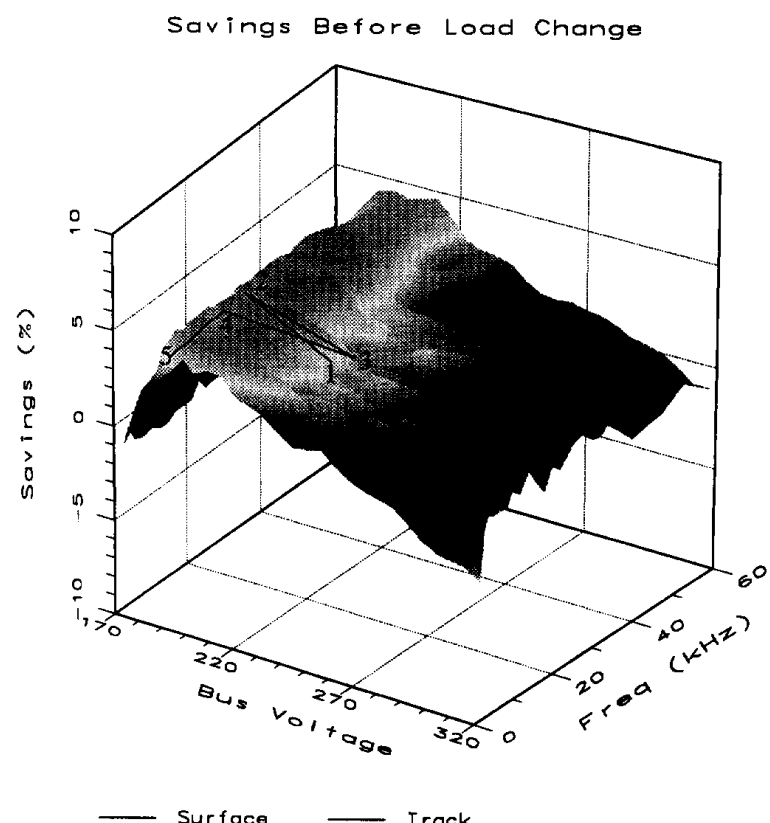

(a)

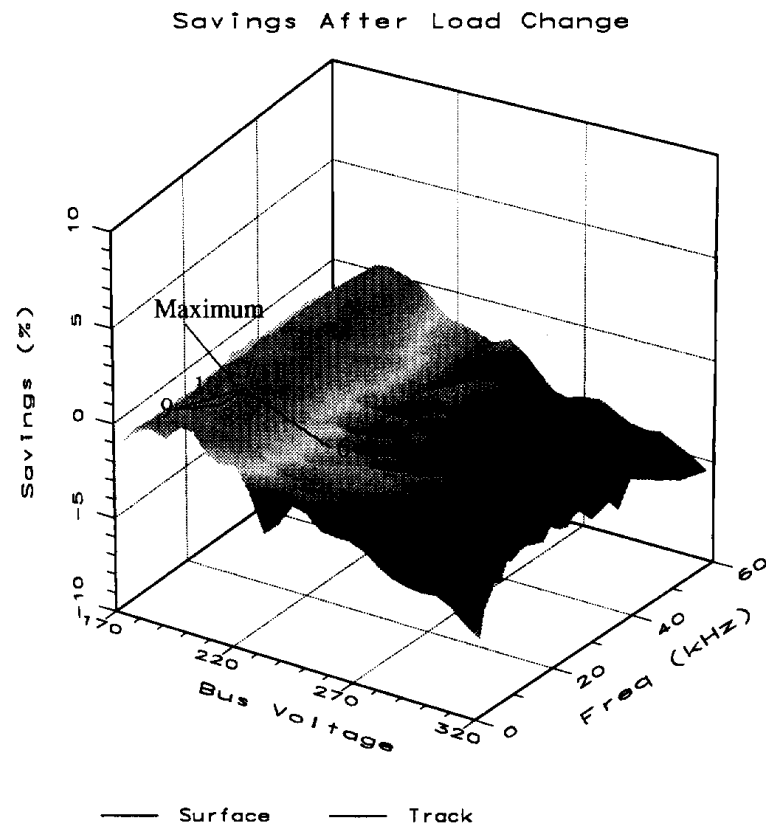

(b)

Fig. 9. Tracking maximum savings under a load change. (a) Track before load change. (b) Track after load change.

The operation of the simplex algorithm due to a load change is illustrated in Fig. 9. Initially the load consists of the single phase bridge rectifier with a capacitive and resistive load drawing 7.2 $A_{\mathrm{RMS}}$. This results in the savings surface shown in Fig. 9(a). The simplex algorithm measures the three starting points 1,2 , and 3 . Based on these three points the simplex

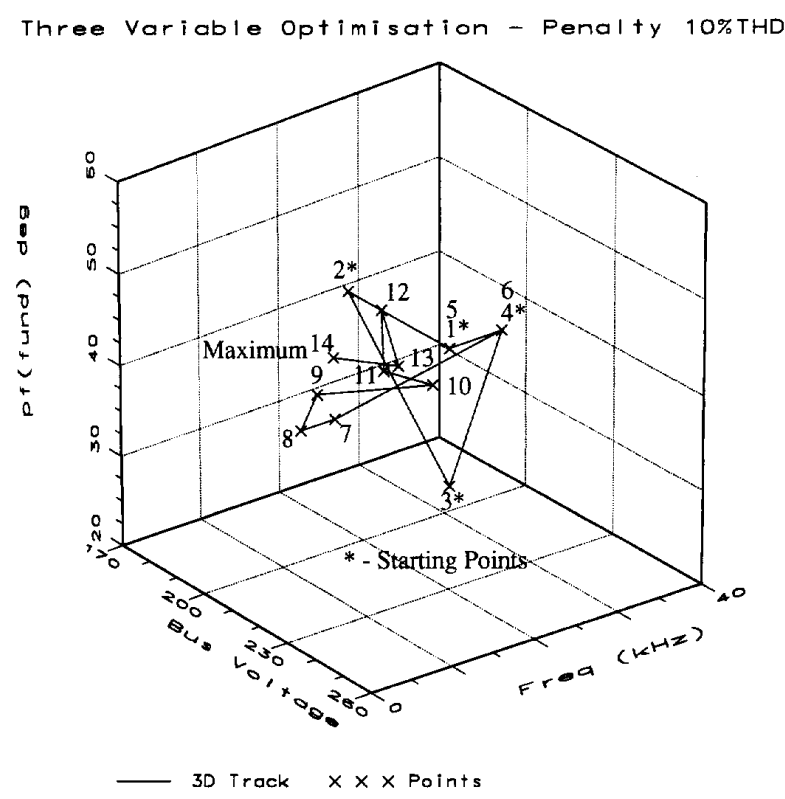

Fig. 10. Three variable optimization track with added penalty-supply current must be less than $10 \%$.

measures a new point 4 and then recalculates and measures another point at 5 . At point 5 the load increases due to an additional resistive ( $3 A_{\mathrm{RMS}}$ ) component being connected in parallel with the bridge rectifier load. The surface now decreases to that shown in Fig. 9(b) and the history terms now have to be remeasured. The three history terms are the three previous highest savings values which were 1,2 , and 4 on Fig. 9(a). The dc bus voltage and switching frequency values of points 1,2 , and 4 correspond to points 6,7 , and 8 on the new savings surface on Fig. 9(b). The simplex algorithm now continues searching until it discovers that the new maximum savings occurs at point 11 .

\section{THREE VARIABLE OPTIMIZATION}

The active filtering system has the ability to manipulate the power amplifier dc bus voltage, average switching frequency, and fundamental power factor to achieve the greatest savings for any particular load. The simplex optimization algorithm is well suited to multidimensional problems [14]. It is difficult to graphically represent a four-dimensional savings surface to show the operation of the optimization algorithm as it steps towards the maximum savings point. The track to the optima is shown in Fig. 10 by displaying the three variables and the tracking sequence between them.

Fig. 10 shows the track leading to the maximum savings point for the load as described in the section on filter performance (Fig. 4) and with the additional penalty that the supply current THD must be less than $10 \%$. The starting point is at a dc bus voltage of $242 \mathrm{~V}$, an average switching frequency of 16 $\mathrm{kHz}$, and fundamental phase displacement of $49^{\circ}$. Three other points are measured and the simplex algorithm then tracks to a maximum savings point of $-21.6 \%$ at $212 \mathrm{~V}, 12 \mathrm{kHz}$, and $44.5^{\circ}$. Introducing a required level of supply current THD to 
be achieved ( $10 \%$ in this case), has resulted in the maximum savings being negative. Thus, it has actually cost money, there are no savings, to reduce the supply current THD from $40.3 \%$ to $9.9 \%$.

\section{CONCLUSIONS}

A relationship for determining the energy savings made from active filtering has been derived. By expressing this savings function as a percentage of the cost of operating the load, it is possible to eliminate the dependency on the real power consumed by the load and the charge for that power. The percentage savings then depends on the energy cost ratio (ratio of charges for real power to apparent power), the phase displacement and THD of the load and supply currents and the efficiency of the active filter system.

Optimization of the energy savings has been implemented with a TMS320C30 DSP intelligent controller using the simplex optimization technique. This simplex method is able to find the optimum operating point, which maximizes savings within a given set of constraints, even under varying load conditions.

\section{REFERENCES}

[1] B. K. Bose, "Recent advances in power electronics," IEEE Trans. Power Electron., vol. 7, no. 1, pp. 2-16, 1992.

[2] J. Arrillaga, D. A. Bradley, and P. S. Bodger, Power System Harmonics. New York: Wiley, 1985.

[3] G. H. Choe and M. H. Park, "A new method for AC harmonic elimination by active power filter," IEEE Trans. Indust. Electron., vol. 35 , no. 1, pp. 141-147, 1988.

[4] H. Akagi, "Trends in active power line conditioners," in Proc. IECON, San Diego, CA, Nov. 9-13, 1992, pp. 19-24.

[5] L. Gyugyi and E. C. Strycula, "Active AC power filters," in Proc. IEEE Indust. Appl. Soc. Annu. Conf., vol. 19-C, 1976, pp. 529-535.

[6] H. Akagi, A. Nabae and S. Atoh, "Control strategy of active power filters using multiple voltage-source PWM converters," IEEE Trans. Indust. Appl., vol. IA-22, no. 3, pp. 460-465, 1986.

[7] J. H. R. Enslin and G. L. van Harmelen, "Real-time, dynamic control of dynamic power filters in supplies with high contamination," in Proc. Power Electron. Specialists Conf., 1990, pp. 887-894.

[8] F. Z. Peng, H. Akagi and A. Nabae, "A new approach to harmonic compensation in power systems-A combined system of shunt passive and series active filters," IEEE Trans. Indust. Appl., vol. 26, no. 6, pp 983-990, 1990.
[9] S. D. Round and R. M. Duke, "A controlled current inverter for active distortion compensation and power factor correction," in Proc. IECON, Kobe, Japan, Oct. 28-Nov. 2, 1991, vol. 1, pp. 735-740.

[10] R. M. Duke and S. D. Round, "The steady state performance of a controlled current active filter." IEEE Trans. Power Electron., Apr. 1993.

[11] — "Novel use of a synthetic sinusoid in the measurement of power system harmonics," in Proc. 6th Conf. Power Electron. and Motion Contr., vol. 2, 1990, pp. 368-373.

[12] P. Papamichalis, Ed., Digital Signal Processing Applications with the TMS320 Family. Houston, TX: Instruments Inc., vol. 3, 1990, pp 31-52.

[13] C. K. Duffey and R. P. Stratford, "Update of harmonic standard IEEE-519: IEEE recommended practices and requirements for harmonic control in electric power systems," IEEE Trans. Indust. Appl., vol. 25 no. 6, pp. 1025-34, 1989.

[14] W. H. Press, B. P. Flannery, S. A. Teukolsky, and W. T. Vetterling, Numerical Recipes the Art of Scientific Computing. Cambridge, MA: Cambridge University Press, 1986.

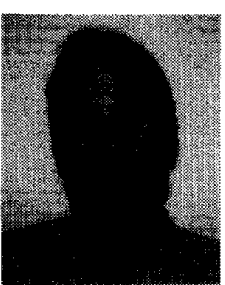

Simon D. Round received the B.E.(Hons) and Ph.D. degrees from the University of Canterbury, Christchurch, New Zealand, in 1989 and 1993, respectively.

On completion of the Ph.D., he held the position of Research Associate in the Department of Electrical Engineering at the University of Minnesota. At the University of Minnesota he investigated the use of neural networks and intelligent controllers for active filters and other power electronic systems. Recently he has been awarded a Research Fellowship by the Royal Norwegian Council for Scientific and Industrial Research. This fellowship will enable him to conduct research in the area of active power filters at the Norwegian Institute of Technology, Trondheim, Norway.

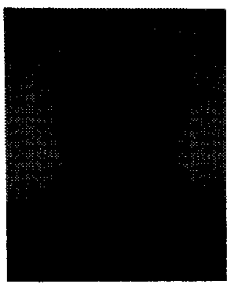

Richard M. Duke received the B.Sc. degree in mathematics, the B.E.(Hons), M.E., and Ph.D. degrees in electrical engineering from the University of Canterbury, Christchurch, New Zealand, in 1965, 1973, 1976, and 1979 respectively.

He worked as an engineer with the New Zealand Electricity Department. Since 1984 he has held a lecturing position at the University of Canterbury. $\mathrm{He}$ is currently a Senior Lecturer in the Department of Electrical and Electronic Engineering. His research interests include power electronics and power system harmonics. 